\title{
Influência de variáveis ambientais sobre a comunidade de oligoquetos (Annelida: Clitellata) em um córrego neotropical
}

\author{
Guilherme Rossi Gorni 1* \\ Roberto da Gama Alves ${ }^{2}$ \\ ${ }^{1}$ Programa de Pós-Graduação em Desenvolvimento Regional e Meio Ambiente \\ Centro Universitário de Araraquara, Rua Voluntários da Pátria, 1309, CEP 14801-320 \\ Araraquara - SP, Brasil \\ ${ }^{2}$ Universidade Federal de Juiz de Fora, Juiz de Fora - MG, Brasil \\ * Autor para correspondência \\ grgorni@gmail.com
}

Submetido em 29/05/2014

Aceito para publicação em 15/12/2014

\section{Resumo}

Dentre os organismos da comunidade bentônica, os oligoquetos são peças fundamentais no fluxo de energia e na reciclagem de matéria orgânica nos ecossistemas aquáticos, onde sua distribuição é influenciada, principalmente, pelos padrões hidrológicos locais. Assim, o objetivo deste estudo foi investigar a influência das variáveis ambientais sobre a comunidade de oligoquetos límnicos em um sistema lótico neotropical. O córrego Galharada está localizado no Parque Estadual de Campos do Jordão (PECJ), no estado de São Paulo, 1.600 $\mathrm{m}$ acima do nível do mar; 192 amostras foram coletadas em quatro pontos selecionados segundo sua ordem hierárquica dentro da bacia hidrográfica (primeira à quarta ordem). A fim de correlacionar as variáveis ambientais e a fauna de oligoquetos utilizou-se a Análise de Correspondência Canônica (CCA). Foram identificadas 16 espécies divididas em quatro famílias (Enchytraeidae, Naididae, Pristinidae e Tubificidae). Os resultados da CCA revelaram que as características físicas dos trechos, como profundidade e largura do córrego, velocidade do fluxo e vazão foram os fatores que mais influenciaram a estrutura da comunidade de oligoquetos aquáticos em Campos do Jordão. As espécies Aulodrilus limnobius e Nais communis foram projetadas junto às variáveis condutividade elétrica e turbidez, indicando possível associação com ambientes enriquecidos com matéria orgânica. A ocorrência de Enchytraeidae, Pristina rosea, Chaetogaster diastrophus, Pristina proboscidea e Limnodrilus hoffmeisteri, aparentemente, não apresentou correlação com as variáveis ambientais analisadas neste estudo. Nossos resultados reforçam a utilização da comunidade de oligoquetos como bioindicadores, participando de processos de avaliação de ecossistemas aquáticos continentais.

Palavras-chave: Análise de Correspondência Canônica; Condições ambientais; Córregos de baixa ordem; Oligoquetos límnicos

\section{Abstract}

Influence of environmental variables on the Oligochaeta (Annelida: Clitellata) community in a neotropical stream. Among the organisms of the benthic community, oligochaetes play a key role in the energy flow and recycling of organic matter in aquatic ecosystems, where their distribution is mainly influenced by local hydrological patterns. Thus, this study aimed to investigate the influence of environmental variables on the community of limnic oligochaetes in a neotropical lotic system. Galharada stream is located within the 
Campos do Jordão State Park (PECJ), in the state of São Paulo, Brazil, 1,600 m above sea level; 192 samples were collected from 4 sites selected according to their hierarchical order within the river basin (first to fourth order). To correlate the environmental variables to the oligochaete fauna, the Canonical Correspondence Analysis (CCA) was performed. We identified 16 species divided into 4 families (Enchytraeidae, Naididae, Pristinidae, and Tubificidae). The results of CCA revealed that the physical characteristics of stretches, such as stream depth and width, flow speed, and flow rate were the factors that most influenced on the structure of the aquatic Oligochaeta community in Campos do Jordão. The species Aulodrilus limnobius and Nais communis were considered along with the variables electrical conductivity and turbidity, indicating a possible association with environments enriched with organic matter. The occurrence of Enchytraeidae, Pristina rosea, Chaetogaster diastrophus, Pristina proboscidea, and Limnodrilus hoffmeisteri was seemingly uncorrelated to the environmental variables analyzed in this study. Our findings reinforce using the Oligochaeta community as bioindicators, by participating in the environmental assessment of continental aquatic ecosystems.

Key words: Canonical Correspondence Analysis; Environmental conditions; Limnic oligochaetes; Low order streams

\section{Introdução}

Rios e córregos podem ser considerados um sistema hierárquico de habitats que diferem em idade, tamanho, e condições ambientais (BEISEL et al., 1998), nas quais podem estar presentes comunidades altamente complexas. Esta heterogeneidade de condições acarreta importantes implicações para a distribuição das comunidades de macroinvertebrados bentônicos (MALMQVIST, 2002; HEINO et al., 2004), atraindo a atenção de inúmeros autores cujas pesquisas abordam diferentes aspectos sobre a distribuição desses organismos (BEISEL et al., 1998; BOYERO; BAILEY, 2001; BURGHERR; WARD, 2001; PALLER et al., 2006, SURIANI et al., 2007; GORNI; ALVES, 2012). Assim, a utilização da comunidade de macroinvertebrados bentônicos apresenta-se como um parâmetro de suma importância para o entendimento da estrutura e funcionamento de ecossistemas lóticos (CUMMINS, 1993).

Dentre os processos que influenciam a distribuição espacial, o acúmulo de matéria orgânica no sedimento é importante, pois serve como fonte de alimento e ponto de apoio para a construção de abrigos. O padrão de abundância de invertebrados límnicos observado em diferentes tipos de substratos pode ser explicado pela variação na qualidade de detritos presentes no substrato (ARUNACHALAM et al., 1991). O fluxo de água também apresenta profunda influência sobre a comunidade bentônica, especialmente em ambientes rasos. Se o movimento da água é fraco, o carbono orgânico pode ser transportado horizontalmente, até alcançar locais de remansos, onde é depositado no fundo propiciando a manutenção de uma rica fauna associada; já as fortes correntes causam drásticas mudanças na estrutura da comunidade (PALMER et al. 1992), podendo inibir o estabelecimento da fauna bentônica (WINTERBOURN; TOWSEND, 1991). Os principais substratos normalmente disponíveis em ambientes lóticos podem ser divididos basicamente em orgânicos e inorgânicos, com maior ou menor quantidade de matéria orgânica, sendo que, os substratos rochosos estão entre os mais estáveis e permanentes, sofrendo menor influência da vegetação ribeirinha e da correnteza (HENRIQUES-OLIVEIRA et al., 2003).

No caso de invertebrados aquáticos que não apresentam vida área em nenhum estágio de seu ciclo biológico, como por exemplo, os Oligochaeta, sua distribuição se restringe mais a aspectos relacionados à dinâmica do ambiente, principalmente os padrões hidrológicos (TAKEDA, 1999; VERDONSCHOT, 2001; NIJBOER et al., 2004; BEHREND et al., 2012 ).

A carência de informações sobre a comunidade de Oligochaeta em ambientes aquáticos neotropicais, bem como a aplicação de espécies nativas como bioindicadores da qualidade dos ecossistemas aquáticos continentais estimularam o desenvolvimento do presente trabalho. Assim, o objetivo do presente estudo foi investigar a relação entre a comunidade de oligoquetos límnicos e variáveis ambientais de um ecossistema lótico neotropical. 


\section{Material e Métodos}

O estudo foi realizado no Parque Estadual de Campos do Jordão (PECJ), localizado ao Norte do município de Campos do Jordão, estado de São Paulo, Brasil (22 $45^{\prime} \mathrm{S}$ e $\left.45^{\circ} 39^{\prime} \mathrm{O}\right)$. Encravado na serra da Mantiqueira, o PECJ abrange uma área de aproximadamente 8172 ha, e apresenta altitude média entre 1600 e $1700 \mathrm{~m}$. O solo do PECJ pertence ao complexo cristalino, formado principalmente por rochas metamórficas (ortognaisse, granito gnaissificado, gnaisse facoidal migmatico, aplito e granulito). A cobertura vegetal do PECJ é composta de Floresta Ombrófila Densa e Mista (Floresta de Araucaria angustifolia) e Campos de Altitude (SEIBERT, 1975). O clima da região, de acordo com a classificação de Köppen, é do tipo Cfb, subtropical de altitude, mesotérmico e úmido, sem estiagem e com temperatura média do mês mais quente inferior a $22^{\circ} \mathrm{C}$ e a precipitação média é de 1800 $\mathrm{mm}$ por ano. $\mathrm{O}$ mês mais chuvoso é janeiro, com mais de $300 \mathrm{~mm}$, e o mais seco é julho, com cerca de $30 \mathrm{~mm}$ (SEIBERT, 1975).

Dentre os ambientes lóticos registrados no parque, foram selecionados para as amostragens quatro pontos do Córrego Galharada, correspondentes à ordem hierárquica dentro da bacia hidrográfica (primeira à quarta ordem). Este córrego tem todo seu curso protegido dentro dos limites do parque, e consiste em um ambiente rico em corredeiras, de leito pedregoso e pouco profundo, com águas frias, límpidas e oxigenadas.

Em todas as coletas, realizadas entre maio de 2005 a abril de 2006, foram aferidas as seguintes variáveis ambientais: teor de oxigênio dissolvido (mg/L), potencial hidrogeniônico $(\mathrm{pH})$, condutividade elétrica $(\mu \mathrm{S} / \mathrm{cm})$, turbidez (NTU), e temperatura da água $\left({ }^{\circ} \mathrm{C}\right)$ com auxilio de sonda multiparâmetro YSI modelo 556 MPS. A profundidade da coluna d'água e a largura dos trechos foram aferidas utilizando-se régua graduada em centímetros (metro). A velocidade da correnteza foi calculada pelo método do flutuador e a vazão através do produto entre a velocidade média da água e a área da coluna d'água.

Os espécimes de Oligochaeta foram colecionados com auxílio de um amostrador de Surber (área de
0, $0361 \mathrm{~m}^{2}$; malha $0,25 \mathrm{~mm}$ ) (MCCAFFERTY, 1981; BICUDO; BICUDO, 2004). Em cada um dos quatro pontos foram realizadas 48 amostragens igualmente distribuídas dentre os principais mesohábitats disponíveis no referido córrego (e.g. Pedra/Corredeira; Folha/Corredeira; Folha/Remanso; Areia/Remanso), perfazendo um total de 192 amostras. O material coletado foi acondicionado em recipientes plásticos e imediatamente fixado em solução de formol $10 \%$. Encaminhadas ao laboratório, as amostras foram triadas sob microscópio estereoscópico. Para a identificação dos oligoquetos foram seguidos critérios taxonômicos adotados por Brinkhurst e Jamieson (1971), Righi (1984), Brinkhurst e Marchese (1989), Pinder e Brinkhurst (1994) e Timm (2009). Exemplares de Tubificidae sexualmente maduros foram diafanizados com lactofenol para a observação das estruturas reprodutoras (BRINKHURST; MARCHESE, 1989). Após identificação os organismos foram acondicionados em frascos com álcool 70\%. A lista de espécies em sinonímia será embasada no catálogo proposto por Christoffersen (2007).

A relação entre as variáveis ambientais e a fauna de oligoquetos foi quantificada pela Análise de Correspondência Canônica (TER BRAAK, 1985), onde os dados abióticos (exceto $\mathrm{pH}$ ) juntamente com os dados faunísticos foram logaritmizados, $\log 10(\mathrm{x}+1)$, a fim de minimizar o efeito dos valores discrepantes. Visando validar a escolha dos eixos da ordenação canônica, bem como verificar a correlação entre a abundância das espécies e a variáveis ambientais analisadas foi utilizado o teste de permutação de Monte Carlo (1000 repetições) $(\alpha=0,05)$. As analises foram realizadas com auxílio do software Multi-variate Statistical Package (MVSP - versão 3.1).

\section{Resultados}

As amostras revelaram um total de 2007 espécimes, sendo inventariados 16 táxons. Dentre estes, foram identificadas 14 espécies, distribuídas em quatro famílias (Enchytraeidae, Naididae, Pristinidae e Tubificidae) (Tabela 1). Apesar de ser representada por apenas um gênero (Pristina), a família Pristinidae foi responsável 
por $50 \%$ dos táxons identificados (oito espécies). Já Naididae apresentou quatro espécies distribuídas em três gêneros (Allonais, Chaetogaster e Nais). A família Tubificidae foi representada por dois gêneros, Aulodrilus e Limnodrilus.

Os resultados da ordenação da CCA consistem na projeção de espécies ao longo de determinados gradientes. Embora tal análise seja multidimensional, somente o primeiro e o segundo eixo (autovalores 0,295 e 0,190 respectivamente) foram incluídos na Figura 1. O teste de permutação de Monte Carlo mostrou que a abundância das espécies as variáveis ambientais foram significativamente correlacionadas $(\mathrm{p}<0,05$, para os dois primeiros eixos).

Grande parcela das relações entre as espécies e as variáveis ambientais foi explicada pelos primeiros dois eixos de ordenações $(29,156 \%$ e $18,781 \%$ respectivamente, totalizando $47,937 \%$ da variação).
O primeiro eixo demonstrou a divisão das variáveis ambientais que caracterizam os pontos amostrados, visto que a parte direita da figura está relacionada a características físicas do córrego, e a parte esquerda por sua vez, está relacionada a características tróficas dos ambientes (ex: condutividade elétrica e turbidez).

Segundo os resultados da CCA, características físicas do ambiente como profundidade, largura do córrego, velocidade do fluxo e vazão apresentamse como fatores importantes na distribuição dos oligoquetos, seguido pela condutividade elétrica, turbidez, temperatura da água e $\mathrm{pH}$. Oxigênio dissolvido teve menor participação na distribuição das espécies. Largura do córrego, velocidade do fluxo, vazão e pH apontaram para a mesma direção. Tais variáveis indicaram ambientes de maior porte podendo ser relacionados, no presente estudo, a trechos do córrego Galharada de quarta ordem (Ponto 4) (quadrante inferior

TABELA 1: Abundância das espécies oligoquetos aquáticos coletadas no período entre maio de 2005 a abril de 2006 no Córrego Galharada, Parque Estadual de Campos do Jordão (PECJ) (SP, Brasil).

\begin{tabular}{|c|c|c|c|c|}
\hline Espécies & Ponto 1 & Ponto 2 & Ponto 3 & Ponto 4 \\
\hline \multicolumn{5}{|l|}{ Enchytraeidae } \\
\hline Enchytraeydae sp.1 & 139 & 450 & 395 & 133 \\
\hline \multicolumn{5}{|l|}{ Naididae } \\
\hline $\begin{array}{l}\text { Allonais paraguayensis paraguayensis (Michaelsen, } \\
\text { 1905) }\end{array}$ & 1 & 0 & 0 & 0 \\
\hline Chaetogaster diastrophus (Gruithuisen, 1828) & 10 & 2 & 7 & 2 \\
\hline Nais communis Piguet, 1906 & 26 & 0 & 5 & 0 \\
\hline Nais variabilis Piguet, 1906 & 0 & 0 & 7 & 0 \\
\hline \multicolumn{5}{|l|}{ Pristinidae } \\
\hline Pristina americana Cernosvitov, 1937 & 0 & 0 & 0 & 2 \\
\hline Pristina biserrata Chen, 1940 & 0 & 1 & 0 & 0 \\
\hline Pristina longiseta Ehrenberg, 1828 & 1 & 22 & 11 & 8 \\
\hline Pristina notopoda Černosvitov, 1937 & 0 & 50 & 0 & 0 \\
\hline Pristina osborni (Walton, 1906) & 7 & 9 & 12 & 5 \\
\hline Pristina proboscidea Beddard, 1896 & 5 & 0 & 2 & 2 \\
\hline Pristina rosea (Piguet, 1906) & 39 & 42 & 43 & 29 \\
\hline Pristina sima (Marcus, 1944) & 4 & 2 & 3 & 2 \\
\hline \multicolumn{5}{|l|}{ Tubificidae } \\
\hline Aulodrilus limnobius Bretscher, 1899 & 489 & 0 & 0 & 0 \\
\hline Limnodrilus hoffmeisteri Claparède, 1862 & 7 & 4 & 0 & 1 \\
\hline
\end{tabular}


FIGURA 1: Diagrama da Análise de Correspondência Canônica da comunidade de Oligochaeta e as variáveis ambientais registradas nos pontos amostrados no córrego Galharada, Campos do Jordão, São Paulo, Brasil. Tágua: Temperatura da água; Odiss: Oxigênio dissolvido; pH; Turb: turbidez; Cond.el: condutividade elétrica; Vel: velocidade do fluxo; Vaz: vazão; Larg: largura do córrego Prof: profundidade do córrego. Alimno: Aulodrilus limnobius; Ncomm: Nais communis; Nvariab: Nais variabilis; Lhoff: Limnodrilus hoffmeisteri; Pprobos: Pristina proboscidea; Cdias: Chaetogaster diastrophus; Psima: Pristina sima; Prose: Pristina rosea; Posborni: Pristina osborni; Pnoto: Pristina notopoda; Plong: Pristina longiseta.

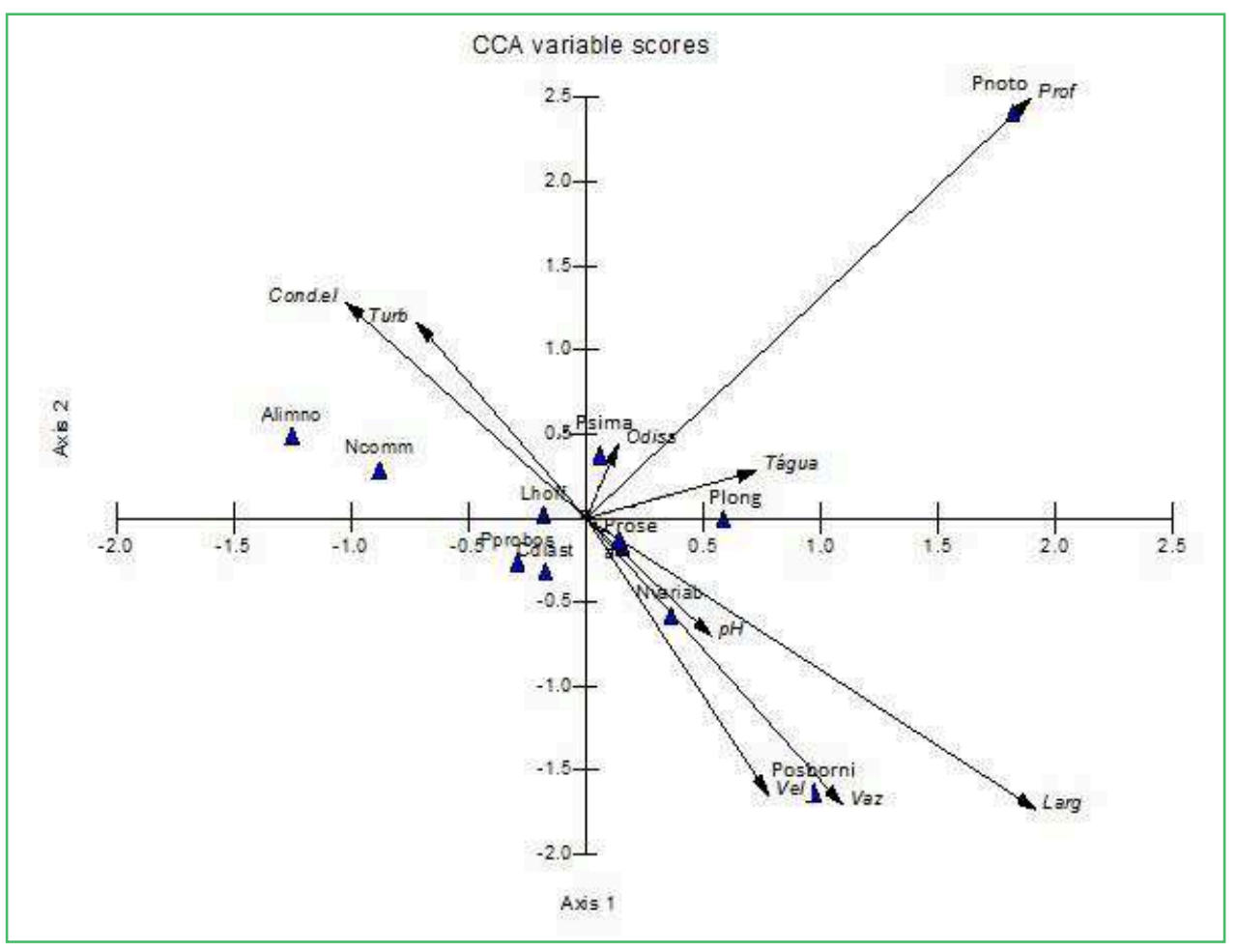

direito - Figura 1), onde as espécies Nais variabilis e Pristina osborni estiveram associadas a essas condições. As variáveis ambientais condutividade elétrica e turbidez apontaram em direção ao lado superior esquerdo da figura, indicando condições de relativo enriquecimento orgânico. As espécies Aulodrilus limnobius e Nais communis foram projetadas neste quadrante, sugerindo uma relação positiva com essas variáveis.

A espécie Pristina notopoda teve correlação positiva com ambientes com maior profundidade. A projeção da espécie Pristina longiseta próximo a variável temperatura da água indica que a presença deste táxon está relacionada a ambientes com temperatura mais elevada. Em contrapartida, Pristina sima demonstrou correlação positiva com o oxigênio dissolvido, indicando preferência por ambientes limpos e oxigenados. O grupo formado por Pristina rosea, Chaetogaster diastrophus, Pristina proboscidea e Limnodrilus hoffmeisteri não apresentou forte relação com as variáveis ambientais aferidas neste trabalho.

\section{Discussão}

O diagrama de ordenação mostrou que os aspectos físicos dos pontos amostrados influenciaram a composição da comunidade de oligoquetos. Estes resultados concordam com estudos de Nijboer et al. (2004), Sanderson et al. (2005), Effenberger et al. (2006) e Bletter et al. (2008), que enfatizam a influência das características físicas do ambiente (profundidade, largura do córrego, velocidade do fluxo e vazão) sobre a estrutura faunística. Estas variáveis influenciam algumas características do córrego como estabilidade do canal, temperatura da água e a presença (ou ausência) do perifiton, que constituem principal restrição à presença de oligoquetos (LENCIONI et al., 2004). Dentre os fatores hidráulicos, a velocidade da correnteza exerce papel chave na distribuição espacial de macrobentos, 
através do carreamento dos organismos rio abaixo (GRZYBKOWSKA et al., 2004). Dumnicka (1994) relata que em córregos de montanhas as espécies de Enchytraeidae amostradas são iguais às registradas nos solos das regiões circundantes, reforçando ainda mais a importância dos fatores hidráulicos (e do processo de "drift") na composição da comunidade dos oligoquetos aquáticos. Martínez-Ansemil e Collado (1996) consideram a velocidade da correnteza juntamente à estabilidade do substrato como os principais fatores que afetam a distribuição espacial das espécies de oligoquetos em ambientes lóticos.

Os processos hidrológicos podem atuar dubiamente na composição das comunidades, uma vez que o aumento do nível da água (dentro de um limite) pode reduzir a probabilidade de dessecação (BEISEL et al., 1998). Esta situação reflete diretamente na estabilidade do ambiente, promovendo maior densidade de invertebrados. Contudo, a elevação excessiva no nível do corpo d'água pode reduzir a iluminação do substrato, afetando negativamente a distribuição das formas bentônicas. Segundo diagrama resultante da CCA, Pristina notopoda aparece como a única espécie associada às condições encontradas em maiores profundidades. Resultados similares foram obtidos por Stacey e Coates (1996), em pesquisa sobre a comunidade de oligoquetos em rios sul-americanos.

Aulodrilus limnobius e Nais communis foram correlacionadas às variáveis ambientais condutividade elétrica e turbidez. Dados de Timm et al. (2001) e Verdonschot (1999; 2001) corroboram essa condição, associando a ocorrência dessas espécies à ambientes com altos valores de matéria orgânica. O táxons Enchytraeidae sp.1, Pristina rosea e L. hoffmeisteri foram projetados no centro do diagrama. L. hoffmeisteri apresenta-se como espécie tolerante e amplamente distribuída, comumente encontrada em ampla distribuição em habitats de corpos d'água de diversos tamanhos (NIJBOER et al., 2004). Essa característica justifica a presença desta espécie no centro do diagrama de ordenação, onde espécies tolerantes e generalistas são posicionadas. Este fato pode ser estendido à família Enchytraeidae, registrada, no presente estudo, em todas as amostras. Dumnicka (1976), Kasprzak e Szczeny
(1976), Dumnicka e Kukula (1990), Lencioni et al. (2004) classificam os enquitreídeos como organismos comuns e abundantes em córregos de baixa ordem (ambientes de montanhas).

Em perspectiva geral, os resultados deste estudo estão em concordância com pesquisas realizadas em córregos de baixa ordem, onde as variáveis físicoquímicas, bem como o regime hidrológico local influenciam diretamente a distribuição das espécies de oligoquetos do córrego Galharada. No entanto, estudos futuros que avaliem a magnitude e o grau de importância da cada variável isoladamente são necessários, uma vez que venham elucidar a profundidade dessas relações. Ademais, nossos resultados reforçam a utilização da comunidade de Oligochaeta como bioindicadores ambientais, atuando como representantes do compartimento biótico na elaboração de novos protocolos de avaliação e conservação de ecossistemas aquáticos continentais.

\section{Agradecimentos}

À Márcia Spies e toda a equipe coordenada pelo Professor Dr. Cláudio Gilberto Froehlich da Universidade de São Paulo (USP-Ribeirão Preto). Ao Programa de Pós-Graduação em Ciências Biológicas (Comportamento e Biologia Animal) - UFJF. Ao Programa Biota - FAPESP pelo auxílio logístico concedido e a CAPES pelo auxilio financeiro.

\section{Referências}

ARUNACHALAM, M.; MADHUSOODANAN NAIR, K. C.; VIJVERBERG, J.; KORTMULDER, K.; SURIYANARAYANAN, H. Substrate selection and seasonal variation in densities of invertebrates in stream pools of a tropical river. Hydrobiologia, Brussels, v. 213, p. 141-148, 1991.

BEHREND, R. D. L.; TAKEDA, A. M.; GOMES, L. C.; FERNANDES, S. E. P. Using oligochaeta assemblages as an indicator of environmental changes. Brazilian Journal of Biology, São Carlos, v. 72, n. 4, p. 873-884, 2012.

BEISEL, J. N.; USSEGLIO-POLATERA, P.; THOMAS, S.; MORETEAU, J. C. Stream community structure in relation to spatial variation: the influence of mesohabitat characteristics. Hydrobiologia, Brussels, v. 389, p. 73-88, 1998.

BICUDO, M.; BICUDO, D. C. Amostragem em Limnologia. São Carlos: RiMa, 2004. 371 p. 
BLETTER, M.; AMSLER, M.; EZCURRA DE DRAGO, I.; MARCHESE, M. Effects of stream hydraulics and other environmental variables on density of Narapa bonettoi (Oligochaeta) in the Paraná River system. River Research and Applications, New Jersey, v. 24, p. 1124-1140, 2008.

BOYERO, L.; BAILEY, R. C. Organization of macroinvertebrates communities at a hierarchy of spatial sacales in tropical stream. Hydrobiologia, Brussels, v. 464, p. 219-225, 2001.

BRINKHURST, R. O.; JAMIESON, B. G. M. Aquatic Oligochaeta of the world. Toronto: University of Toronto Press, 1971. $860 \mathrm{p}$.

BRINKHURST, R. O.; MARCHESE, M. R. Guia para la indentificacion de Oligoquetos aquáticos continentales de Sud y Centroamerica. Santa Fé: Climax, 1989. 207 p.

BURGHERR, P.; WARD, J. V. Longitudinal and seasonal distribution patterns of the benthic fauna of an alpine glacial stream (Val Roseg, Swiss Alps). Freshwater Biology, Oxford, v. 42, n. 12, p. 1705-1721, 2001.

CHRISTOFFERSEN, M. L. A catalogue of aquatic microdile oligochaetes (Annelida: Clitellata) from South America. Acta Hydrobiologica Sinica, Wuhan, v. 31, p. 59-86, 2007.

CUMMINS, K. W. Invertebrates. In: CALOW, P.; PETTS, G. E. (Ed.). The rivers handbook - Hydrological and ecological principles. 2. ed. Oxford: Blackwell Science Ltd., 1993. p. 234-250.

DUMNICKA, E. Oligochaetes (Oligochaeta) of some streams of the High Tatra Mts ando f the River Balka Tatrzańska. Acta Hydrobiologica, Cracóvia, v. 18, n. 3, p. 305-315, 1976.

DUMNICKA, E. Communities of oligochaetes in mountain streams of Poland. Hydrobiologia, Brussels, v. 278, p. 107-110, 1994.

DUMNICKA, E.; KUKULA, K. The communities of oligochaetes of the Wolosatka and Terebowiec streams the Bieszczady National Park, southeastern Poland. Acta Hydrobiologica, Cracóvia, v. 30, n. 3/4, p. 423-435, 1990.

EFFENBERGER, M.; SAILER, G.; TOWNSEND, C. R.; MATTHAEI, C. D. Local disturbance history and habitat parameters influence the microdistribution of stream invertebrates. Freshwater Biology, Oxford, v. 51, p. 312-332, 2006.

GORNI, G. R.; ALVES, R. G. Oligochaetes (Annelida, Clitellata) in a neotropical stream: a mesohabitat approach. Iheringia, Série Zoologia, Porto Alegre, v. 102, n. 1, p. 106-110, 2012.

GRZYBKOWSKA, M.; DUKOWSKA, M.; FIGIEL, K.; SZCZERKOWSKA, E.; TSZYDEL, M. Dynamics of macroinvertebrate drift in a lowland river. Zoologica Poloniae, Varsóvia, v. 49, n. 1-4, p. 111-127, 2004.

HEINO, J.; LOUHI, P.; MUOTKA, T. Identifying the escales of variability in stream macroinvertebrate abundance, functional composition and assemblage structure. Freshwater Biology, Oxford, v. 49, p. 1230-1239, 2004.

HENRIQUES-OLIVEIRA, A. L.; DORVILLÉ, L. F. M.; NESSIMIAN, J. L. Distribution of Chironomidae larvae fauna (Insecta: Diptera) on different substrates in a stream Floresta da Tijuca, RJ, Brazil. Acta Limnologica Brasiliensia, Botucatu, v. 15, p. 69-84, 2003.

KASPRZAK, K.; SZCZENY, B. Oligochaetes (Oligochaeta) of the River Raba. Acta Hydrobiologica, Cracóvia, v. 18, n. 1, p. 75-87, 1976.

LENCIONI, V.; DUMNICKA, E.; MAIOLINI, B. The oligochaete fauna in high mountain streams (Trentino, NE Italy): ecological and taxonomical remarks. Studi Trentini di Scienze Naturali Acta Biologica, Trento, v. 81, p. 167-176, 2004.

MALMQVIST, B. Aquatic invertebrates in riverine landscapes. Freshwater Biology, Oxford, v. 47, p. 679-694, 2002.

MARTÍNEZ-ANSEMIL, E.; COLLADO, R. Distribution patterns of aquatic oligochaetes inhabiting watercouses in Northwestern Iberian Península. Hydrobiologia, Brussels, v. 334, p. 73-83, 1996.

MCCAFFERTY, W. P. Aquatic Entomology: the fisherman's and ecologist's illustrated guide to insects and their relatives. Boston: Science Books International, 1981. 448 p.

NIJBOER, R. C.; WETZEL, M. J.; VERDONSCHOT, P. F. M. Diversity and distribution of Tubificidae, Naididae, and Lumbriculidae (Annelida: Oligochaeta) in the Netherlands: na evaluation of twenty years of monitoring data. Hydrobiologia, Brussels, v. 520, p. 127-141, 2004.

PALMER, M. A.; BELY, A. E.; BERG, K. E. Response of invertebrates to lotic disturbance: a test of the hyporheic refuge hypothesis. Oecologia, Berlin, v. 89, p. 182-194, 1992.

PALLER, N. H.; SPECHT, W. L.; DYER, S. A. Effects of stream size on taxa richness and other commonly used benthic bioassesment metrics. Hydrobiologia, Brussels, v. 568, p. 309-316, 2006.

PINDER, A. P.; BRINKHURST, R. O. A preliminary guide to the identification of the microdrile Oligochaeta of Australian Inland Waters. Albruy: Cooperative Research Center for Freshwater Ecology, 1994. 137 p.

RIGHI, G. Manual de identificação de invertebrados límnicos do Brasil. Brasília: CNpq/Coordenação Editorial, 1984. 48 p.

SANDERSON, R. A.; EYRE, M. D.; RUSHTON, P. The influence of stream invertebrate composition at neighbouring sites on local assemblage composition. Freshwater Biology, Oxford, v. 50, p. 221-231, 2005.

SCHROEDER-ARAUJO, L. T.; STEMPNIEWSKI, H. L.; CIPÓLlI, M. N.; SANTOS, L. E.; SANTO-PAULO, M.; CORREA-CREMONESI, W. Estudo limnológico e climatológico da região do Parque Estadual de Campos do Jordão, SP, com vistas ao povoamento com truta arco-íris, Salmo irideus Gibbons. Boletim de Instituto de Pesca, São Paulo, v. 13, n. 2, p. 63-76, 1986.

SEIBERT, P. Plano de manejo do Parque Estadual de Campos do Jordão. Boletim Técnico do Instituto Florestal, São Paulo, v.19, p. 1-153, 1975.

STACEY, D. F.; COATES, K. A. Oligochaetes (Naididae, Tubificidae, Opistocystidae, Enchytraeidae, Sparganophilidae and Alluroididae) of Guyana. Hydrobiologia, Brussels, v. 334, p. $17-$ 29, 1996.

SURIANI, A. L.; FRANÇA, R. S.; PAMPLIN, PAZ.; MARCHESE, M.; LUCCA, J. V.; ROCHA, O. Species richness and distribution of oligochaetes in six reservoirs on Middle and Low Tietê River (SP, Brazil). Acta Limnologica Brasiliensia, Botucatu, v. 19, n. 4, p. 415-426, 2007.

TAKEDA, A. M. Oligochaeta community of alluvial Upper Paraná River, Brazil: spatial and temporal distribution (1987-1988). Hydrobiologia, Brussels, v. 412, p. 35-42, 1999.

TER BRAAK, C. J. Correspondence analysis of incidence and abundance data: properties in terms of a unimodal response model. Biometrics, Malden, v. 41, p. 859-873, 1985. 
TIMM, T. A guide to the freshwater Oligochaeta and Polychaeta of Northern and Central Europe. Lauterbornia, Dinkelscherben, v. 66, p. 1-235, 2009.

TIMM, T.; SEIRE, A.; PALL, P. Half a century of oligochaete research in Estonian running waters. Hydrobiologia, Brussels, v. 463, p. 223-234, 2001.

VERDONSCHOT, P. F. M. Micro-distribution of oligochaetes in a soft-bottomed lowland stream (Elsbeek; Netherlands). Hydrobiologia, Brussels, v. 406, p. 149-163, 1999.

VERDONSCHOT, P. F. M. Hydrology and substrates: determinants of oligochaete distribution in lowland streams (The Netherlands).

Hydrobiologia, Brussels, v. 463, p. 249-262, 2001.

WINTERBOURN, M. J.; TOWSEND, C. R. Streams and River: one-way flow system. In: BARNES, R. S. K.; MANN, K. H (Ed.). Fundamentals of aquatic ecology. 2 ed. Oxford: Blackwell Scientific Publ., 1991. p. 230-242. 